\title{
Pedagogía ambiental y didáctica ambiental como fundamentos del currículo para la formación ambiental
}

\author{
JULIO CÉSAR TOVAR-GÁLVEZ \\ Universidad Antonio Nariño, Bogotá, Colombia
}

\section{INTRODUCCIÓN}

El estudio que se presenta en este documento se encuentra enmarcado en el proyecto de investigación Hacia un currículo para la formación ambiental en la educación superior colombiana (financiado por la Vicerrectoría de Ciencia-Tecnología e Innovación de la Universidad Antonio Nariño, vigencia 2011-2013), el cual tiene como principal objetivo "proponer lineamientos curriculares pertinentes para la formación ambiental en el contexto de la educación superior colombiana" (Grupo Conciencia, 2011).

Para lograr tal objetivo, ha sido necesario hacer algunas delimitaciones que permitan abordar el tema del currículo; en tal sentido, el presente artículo se centra en la problemática relativa a lo que se ha venido construyendo sobre la pedagogía ambiental y sobre la didáctica ambiental, entendidos éstos dos conceptos como unos de los pilares para aproximarse al currículo para la formación ambiental.

Antes de llegar al currículo, la construcción del objeto de estudio "pedagogía ambiental y didáctica ambiental", es en sí mismo un problema que suscita diversas preguntas, anteponiendo la relatividad sobre las propuestas que sobre el mismo se hagan, en la medida en que los cuestionamientos pueden llevar a respuestas muy amplias. Consecuentemente es necesario preguntar: ¿qué es lo pedagógico?, ¿qué 
es lo didáctico?, ¿qué es la formación ambiental?, ¿se puede hablar de pedagogía ambiental?, y ¿se puede hablar de didáctica ambiental?, entre otras.

Para avanzar en la solución de dichos cuestionamientos, el artículo se estructura así:

a) planteamiento y delimitación del problema entorno a la pedagogía ambiental y la didáctica ambiental, como pilares para la fundamentación de un currículo para la formación ambiental;

b) proposición de un marco teórico con dos partes: i) una sobre lo pedagógico y lo didáctico, de manera que se conceptualicen como categorías generales de análisis, ii) la otra sobre qué es un estado del arte;

c) elaboración del estado del arte, para identificar las tendencias teóricoprácticas que existen sobre pedagogía ambiental y sobre didáctica ambiental.

\section{PROBLEMA DE INVESTIGACIÓN}

Hablar o adentrarse en el tema del currículo es una compleja tarea, en tanto, a través de la historia, el currículo ha sido conceptualizado y vivido desde diferentes perspectivas; por lo que es incierto apuntar a una u otra teoría de manera determinante. Al parecer, el currículo está ligado con unas u otras posturas filosóficas, epistemológicas, sociológicas, pedagógicas y didácticas, lo que muestra la amplitud de concepciones sobre currículo. Sin embargo, algunos expertos han venido acordando, más o menos, una clasificación amplia de las teorías curriculares:

a) una ligada a los fundamentos del positivismo, que asume el currículo como una forma explicar y predecir el horizonte y dinámica de los procesos educativos, a través del control y el establecimiento de relaciones directas entre objetivos y métodos;

b) una centrada en supuestos del paradigma hermenéutico, que ya no se centra en el conocimiento como objetivo de la escuela, sino en las necesidades de los sujetos y de los contextos;

c) y otra ubicada en los fundamentos de la teoría crítica, que no establece disyunción entre el objetivo del saber y el objetivo de responder a los contextos, sino que plantea una dialéctica en la que el sujeto es quien construye el conocimiento en busca de su liberación de la opresión (a manera de ejemplo, se cita a: Ferrández, 1991; Guerra, 2008; Pacheco, 2001; Román; Díez, 2003).

De lo anterior se puede aseverar entonces que, entre varios aspectos para fundamentar una concepción y práctica de currículo, es necesario tomar una postura frente a lo pedagógico y a lo didáctico. Por extensión, para pensar la formación ambiental en la educación superior, es necesario fundamentar el currículo, tomando una postura desde la pedagogía ambiental y la didáctica ambiental. Sin embargo, 
surgen grandes preguntas que abren el amplio campo de investigación en torno al currículo para la formación ambiental: ¿qué es pedagogía y qué es didáctica?, lo que consecuentemente permite preguntar ¿qué es pedagogía ambiental y qué es didáctica ambiental?

Al delimitar la problemática del currículo para la formación ambiental, a la cuestión de la problemática ambiental y la didáctica ambiental, a través de las tres preguntas formuladas, el artículo se plantea: a) abordar el tema de lo pedagógico y de lo didáctico, $\mathrm{y} b$ ) establecer lo pedagógico y lo didáctico como categorías de análisis para identificar las tendencias sobre pedagogía ambiental y didáctica ambiental en literatura especializada.

\section{MARCO TEÓRICO}

\section{LO PEDAGÓGICO Y LO DIDÁCTICO}

Como punto de partida es necesario decir que existen múltiples interpretaciones sobre la naturaleza de la pedagogía, siendo ligada a diversas disciplinas o campos del saber. Sin embargo, a pesar de la constante transformación de los discursos y de los enfoques de los debates, es posible identificar avances y ciertos consensos; por ejemplo, Zaccagnini (2003) señala que los modelos pedagógicos históricos por lo menos deben ser identificados por núcleos paradigmáticos, bases filosóficas, bases histórico-sociales, bases psicológicas y modelos didácticos, pudiéndose así hacer un estudio complejo de las transformaciones de la pedagogía respecto a los cambios en la conceptualización de su naturaleza, su finalidad, su estructura y dinámica.

Desde la mirada epistemológica, Díaz (2005) hace un estudio en el que señala que el conocimiento pedagógico se refiere al conocimiento (valores, ideologías, actitudes y prácticas) que es producido por el docente en su interacción con otros sujetos en la dialéctica del aula, a través de la transformación de los significados del proceso educativo, ya sea hecho de manera explícita o implícita; y, por su parte, Velázquez (2005) ubica a la pedagogía como una disciplina emergente, cuyo problema de estudio es el fenómeno educativo en todas sus dimensiones.

De alguna manera, lo anterior muestra que lo pedagógico hace referencia a formas de entender o teorías sobre la educación, que es un proceso desde el que genera conocimiento sobre los procesos educativos, y que es la vivencia de los procesos educativos; entendiendo que dichas teorías, formas de producción y vivencias son relativas y provisionales, en tanto su objeto de estudio o razón de ser, la educación, está en constante transformación por su complejidad.

Por otro lado, el de lo didáctico, es posible identificar al menos dos grandes referentes (García, 2012; Tovar-Gálvez) b): a) la didáctica general fundamentada en los principios de las teorías del aprendizaje, y b) las didácticas específicas, las cuales se centran en los presupuestos históricos y epistemológicos de cada disciplina. Sin dejar de lado la relevancia de los aportes de la didáctica general, es necesario reconocer la relevancia que están adquiriendo las didácticas específicas, teniendo 
como indicador las múltiples revistas especializadas en cada una, así como programas pos-graduales que forman investigadores en tales áreas.

$\mathrm{E} 1$ caso más notable es el de la didáctica de las ciencias naturales, puesto que existe extensa literatura especializada sobre la argumentación de su estatuto científico y su carácter parcialmente autónomo (Adúriz-Bravo; Izquierdo, 2002; Cachapuz et al., 2001; Gallego-Badillo, 2004; Tovar-Gálvez, 2008; 2009); así mismo, se ha venido fundamentando la didáctica de las matemáticas (Fernández, 2000; Godino, 2010; 2011; Moreno, 2007; Zambrano, 2004), más recientemente la didáctica de las ciencias sociales (Aisenberg, 1998; Pagés, 1994; Prats, 2000; 2003) y la didáctica las lenguas (Díaz, 1996; Núñez et al., 2008; Trujillo, 2005; Vez, 2004; Zanón, 2007); en tanto sus naturalezas, sus objetos de estudio, sus cuerpos de conocimiento y metodologías de producción, sus comunidades y lenguajes propios.

Desde las discusiones y propuestas de los autores citados anteriormente, es posible decir que la didáctica, entendida como una disciplina, tiene como objeto de estudio el proceso de enseñanza-aprendizaje-evaluación de los modelos teórico-práctico-valorativos de las disciplinas, alrededor de lo cual se han venido desarrollando líneas de investigación que problematizan dicho fenómeno y desde la cuales se han construido referentes teórico y metodológicos, así como consolidación de comunidades internacionales de expertos. Ahora bien, lo didáctico como vivencia, hace referencia a las interacciones que buscan que se de la enseñanza-aprendizaje-evaluación de las disciplinas.

\section{EL ESTADO DEL ARTE}

La construcción del estado del arte es un proceso que consiste en revisar y analizar qué se ha hecho en un campo de investigación o frente a un tema, de tal manera que se pueda tener una visión amplia sobre las tendencias teóricas y metodológicas, así como las falencias, incongruencias y/u oportunidades reportadas en la literatura especializada. Con el estado del arte el investigador conoce el tema y las formas en que se ha venido abordando; ello no quiere decir que el investigador se ciña a las propuestas teóricas y metodológicas de una tradición académica, sino que puede significarle la oportunidad de proponer alternativas.

Es importante señalar que no se trata de una sucesión de resúmenes, sino una construcción que haga un análisis de lo reportado y un meta-análisis en el que se dé cuenta de la forma en que se ha venido produciendo en el área, para lo que se puede responder a tres preguntas básicas: ¿cuáles son las principales tendencias teóricas a través de las cuales se interpreta el problema?, ¿cuáles son las principales tendencias o propuestas metodológicas a través de las cuales se aborda el problema?, ¿cuáles son las principales conclusiones a las que han llegado?

Tampoco se trata de solo presentar lo que dice la literatura, sino que el investigador debe manifestar su postura frente a ello y sobre todo ver cómo le aporta a su proyecto. Finalmente lo que van a responderse es: ¿̇cuál o culés son las propuestas teóricas más convenientes para comprender el tema o el problema que me interesa abordar?, ¿cuál o cuáles son las principales metodologías que son 
acordes para abordar el tema o problema de interés?; sin embargo el asunto es no llegar a la rigidez o a la elección de una sola postura; puede que se tomen elementos de uno u otro enfoque y propuestas metodológicas, pero sobre todo, es posible que sea necesario proponer alternativas, coherentes y argumentadas.

\section{METODOLOGÍA}

Esta sección es presentada y desarrollada con el objetivo de responder a la pregunta ¿qué es pedagogía ambiental y qué es didáctica ambiental?, a través de la elaboración de un estado del arte, teniendo como categorías de análisis lo pedagógico y lo didáctico. La construcción del estado del arte a partir de las categorías permite identificar las tendencias sobre pedagogía ambiental y sobre didáctica ambiental.

A partir de lo dicho, se establece a continuación un proceso interpretativo de la literatura especializada sobre propuestas teóricas y/o sobre experiencias en torno a lo formativo ambiental. Para esto, primero se hace una explicación sobre qué es un estado del arte, a continuación se presenta la metodología para construir un estado del arte (definición de categorías, descripción de la gestión de la información, descripción del análisis de la información), para finalmente mostrar el análisis de la información (resultados de la gestión de la información, resultados de la agrupación de información para identificar las tendencias).

\section{CATEGORÍAS DE ANÁLISIS PARA EL ESTUDIO}

Teniendo en cuenta que parte del objetivo es fundamentar el currículo para la formación ambiental, no es posible tener una postura a priori sobre los mismos conceptos "pedagogía ambiental" y "didáctica ambiental", por lo que se propone hacer una lectura e interpretación de la literatura especializada desde las orientaciones generales sobre lo que se puede entender por pedagogía y por didáctica. De esta manera, para tener una referencia en la construcción del estado del arte y proponer fundamentos sobre pedagogía ambiental y sobre didáctica ambiental, se establecen las siguientes categorías o criterios que permiten hacer la lectura, discriminación y análisis de los referentes y la construcción de la propuesta final:

\subsection{Categoría pedagogía}

Se construye por las reflexiones en torno a aspectos como: a) concepción de educción, abordándose así lo filosófico y lo epistémico; b) rol de los actores en el proceso educativo, implicando preguntar por aspectos sociológicos y psicológicos; c) construcción de los perfiles-sujetos a través de los procesos educativos, haciendo referencia esto a lo curricular. En términos prácticos, dichos tres aspectos serán los criterios de análisis del contenido de las fuentes de información, para así obtener lo que cada referente conceptualiza, implícita o explícitamente, sobre pedagogía ambiental. 


\subsection{Categoría didáctica}

Se construye por las reflexiones en torno a aspectos como:

a) cómo se entiende el proceso de aprendizaje;

b) cómo se entiende la enseñanza;

c) cómo se dan los procesos de enseñanza-aprendizaje;

d) evaluación de los procesos de enseñanza aprendizaje.

Para la elaboración del estado del arte, los cuatro aspectos definidos permiten identificar la concepción que sobre didáctica ambiental presentan los referentes a analizar.

Ahora bien, es posible que la información encontrada en las fuentes de información no se refiera directa y explícitamente a las categorías y a los aspectos que les definen; de ahí que sea un trabajo interpretativo. Así mismo, la variación en las formas en que se expresan o de finen las sub-categorías o literales en cada referente, son las que marcarán las tendencias en cuanto a lo que se puede llamar "pedagogía ambiental” y "didáctica ambiental".

\section{GESTIÓN DE LA INFORMACIÓN}

Este proceso consiste en la búsqueda de fuentes de información especializada, para a partir de las mismas obtener gran variedad de documentos. Con la existencia de documentos se hace una valoración que permita discriminar y organizar la información, en función de su utilidad para los intereses del estudio a realizar. Así, para el caso de lo presentado en este artículo, se hace revisión en varias bases de datos digitales de libre acceso, se revisan revistas digitales especializadas, así como documentos de normativa sobre educación ambiental; en varios idiomas. Finalmente, se seleccionan y clasifican aquellos documentos que hacen referencia o que contienen información que al ser analizada a través de la conceptualización de las categorías, permiten identificar elementos teóricos y prácticos sobre la pedagogía ambiental y sobre la didáctica ambiental.

\section{ANÁLISIS DE LA INFORMACIÓN}

\subsection{Descripción de los fundamentos}

Esta parte de la construcción del estado del arte consiste en la fase hermenéutica, en la que se interpretan y presentan los argumentos que de la literatura especializad y demás fuentes consultadas, permiten la identificación de las sub-categorías o elementos que definen las categorías "pedagogía ambiental" y "didáctica ambiental". 
Cuadro 1 - Descripción de fundamentos teóricos por cada artículo

\begin{tabular}{|l|l|l|}
\hline \multicolumn{1}{|c|}{ Referente } & Fundamentos pedagógicos & \multicolumn{1}{c|}{$\begin{array}{c}\text { Fundamentos didáctica } \\
\text { ambiental }\end{array}$} \\
\hline Referencia 1 & Texto relativo a pedagogia ambiental & Texto relativo a didáctica ambiental \\
\hline Referencia $\mathrm{n}$ & Texto relativo a pedagogia a mbiental & Texto relativo a didáctica ambiental \\
\hline
\end{tabular}

Fonte: Investigación del autor.

\subsection{Análisis comparado y tendencias}

Esta fase consiste en hacer una contrastación entre los referentes identificados durante la etapa anterior. Dicha confrontación teórica permite identificar tendencias conceptuales y metodológicas, así como los posibles aspectos que aún requieren mayor discusión y debate. Este análisis el que permite ver las perspectivas de construcción e investigación frente al problema de investigación y es desde allí donde se construyen los argumentos para hacer propuestas. El análisis se puede orientar a través de la respuesta a la pregunta ¿cuáles son las tendencias teóricas en la forma de comprender lo pedagógico ambiental y lo didáctico ambiental?

\section{RESULTADOS Y ANÁLISIS}

\section{GESTIÓN DE LA INFORMACIÓN}

\subsection{Selección de las fuentes de información}

Se realizó una búsqueda a través de varios medios digitales. Así, se consultó la base de datos Directory of Open Access Journals (www.doaj.org), la cual pertenece a la Universidad de Lund en Suecia y cuenta con el apoyo y patrocinio de: Open Society Institute, National Library of Sweden, Scholarly Publishing and Academic Resources Coalition (SPARC), SPARC Europe, Axiell, EBSCO, INASP y la Swedish Library Association. Para el 20 de agosto de 2011, cuando se realizó la principal revisión, la base de datos filtraba información de 6.960 revistas y un total de 624.093 artículos.

La combinación de criterios para la búsqueda se dio como se muestra el cuadro a continuación y los resultados fueron depurados en función del interés del estudio a realizar.

Cuadro 2 - Palabras clave combinadas en la base de datos y artículos obtenidos

\begin{tabular}{|l|l|}
\hline $\begin{array}{l}\text { All Fields = pedagogía AND } \\
\text { All Fields = ambiental }\end{array}$ & $\begin{array}{l}7 \text { documentos: } 4 \text { en español y } 3 \text { en portugués; por el } \\
\text { título y revisión del resumen, } 6 \text { aportan a la temática } .\end{array}$ \\
\hline $\begin{array}{l}\text { All Fields = universidad AND } \\
\text { All Fields = ambiental }\end{array}$ & $\begin{array}{l}83 \text { documentos: todos en español; por el título y } \\
\text { revisión del resumen, } 13 \text { aportan a la temática. }\end{array}$ \\
\hline
\end{tabular}

(continua...) 
(...continuação)

\begin{tabular}{|l|l|}
\hline $\begin{array}{l}\text { All Fields = didáctica AND } \\
\text { All Fields = ambiental }\end{array}$ & $\begin{array}{l}10 \text { documentos: } 8 \text { en español, } 1 \text { en portugués y } 1 \text { en catalán; } \\
\text { por el título y revisión del resumen, } 7 \text { aportan a la temática. }\end{array}$ \\
\hline $\begin{array}{l}\text { All Fields = educación AND } \\
\text { All Fields = ambiental }\end{array}$ & $\begin{array}{l}144 \text { documentos: por el título y revisión del } \\
\text { resumen, } 68 \text { aportan a la temática. }\end{array}$ \\
\hline $\begin{array}{l}\text { All Fields = universidade AND } \\
\text { All Fields = ambiental }\end{array}$ & $\begin{array}{l}55 \text { documentos: por el título y revisión del } \\
\text { resumen, } 7 \text { aportan a la temática. }\end{array}$ \\
\hline $\begin{array}{l}\text { Title }=\text { university AND } \\
\text { Title }=\text { environmental }\end{array}$ & $\begin{array}{l}17 \text { documentos: } 8 \text { en inglés, } 2 \text { en chino, } 2 \text { en portugués, } \\
2 \text { en español, } 2 \text { en turco y } 1 \text { en eslovaco; por el título } \\
\text { y revisión del resumen, } 11 \text { aportan a la temática. }\end{array}$ \\
\hline $\begin{array}{l}\text { All Fields = pedagogy AND } \\
\text { All Fields = environmental }\end{array}$ & $\begin{array}{l}11 \text { documentos: } 8 \text { en inglés, } 2 \text { en español y } 1 \text { en portugués; } \\
\text { por el título y revisión del resumen, } 4 \text { aportan a la temática. }\end{array}$ \\
\hline $\begin{array}{l}\text { Title }=\text { teaching AND } \\
\text { Title }=\text { environmental }\end{array}$ & $\begin{array}{l}7 \text { documentos: todos en inglés; por el título y } \\
\text { revisión del resumen, } 3 \text { aportan a la temática. }\end{array}$ \\
\hline
\end{tabular}

Fonte: www.doaj.org

Así mismo se optó por hacer una revisión de otras fuentes que por tradición publican material referente al tema de la educación ambiental y afines:

\section{Cuadro 3 - Artículos obtenidos por revisión en otras fuentes especializadas}

\begin{tabular}{|l|l|}
\hline $\begin{array}{l}\text { Biblioteca Digital del Centro de Recursos Documentales e } \\
\text { Informáticos de la OEI, recursos por materia “Educación } \\
\text { Ambiental”, <http://www.oei.es/oei-credi/materias/lista/12> }\end{array}$ & $\begin{array}{l}163 \text { registros: } 145 \text { en español, } 17 \text { en } \\
\text { portugués y } 1 \text { en catalán; por el título, } \\
\text { 24 registros aportan a la temática. }\end{array}$ \\
\hline $\begin{array}{l}\text { International Journal of Environmental and Science } \\
\text { Education, Turkey, <http://www.ijese.com/index.htm> }\end{array}$ & $\begin{array}{l}\text { Por el título y revisión del resumen, } \\
21 \text { artículos aportan a la temática. }\end{array}$ \\
\hline $\begin{array}{l}\text { Revista Luna Azul, de la Universidad de Caldas, } \\
\text { Colombia, <http://lunazul.ucaldas.edu.co/> }\end{array}$ & $\begin{array}{l}\text { Por el título y revisión del resumen, } \\
18 \text { artículos aportan a la temática. }\end{array}$ \\
\hline $\begin{array}{l}\text { Revista Eureka sobre Enseñanza y Divulgación de } \\
\text { las Ciencias, de la Universidad de Cádiz, España, } \\
<\text { http://reuredc.uca.es/index.php/tavira> }\end{array}$ & $\begin{array}{l}\text { Por el título y revisión del resumen, } \\
9 \text { artículos aportan a la temática. }\end{array}$ \\
\hline $\begin{array}{l}\text { (Revista) Utopía y Praxis Latinoamericana, de } \\
\text { la Universidad de Zulia, Venezuela,<http:// } \\
\text { revistas.luz.edu.ve/index.php/upl/index> }\end{array}$ & $\begin{array}{l}\text { Por el título y revisión del resumen, } \\
7 \text { artículos aportan a la temática. }\end{array}$ \\
\hline $\begin{array}{l}\text { International Electronic Journal of } \\
\text { Environmental Education,Turkey, <http://www. } \\
\text { iejeegreen.com/index.php/iejeegreen> }\end{array}$ & $\begin{array}{l}\text { Por el título y revisión del resumen, } \\
7 \text { artículos aportan a la temática. }\end{array}$ \\
\hline $\begin{array}{l}\text { Revista Ciência em Extensão, Universidade Estadual } \\
\text { Paulista “Júlio de Mesquita Filho", Brasil, <http:// } \\
\text { ojs.unesp.br/index.php/revista_proex/index> }\end{array}$ & $\begin{array}{l}\text { Por el título y revisión del resumen, } \\
6 \text { artículos aportan a la temática. }\end{array}$ \\
\hline $\begin{array}{l}\text { (Revista) Ecosistemas, de la Asociación Española de } \\
\text { Ecología Terrestre, <http://www.revistaecosistemas.net/> }\end{array}$ & $\begin{array}{l}\text { Por el título y revisión del resumen, } \\
6 \text { artículos aportan a la temática. }\end{array}$ \\
\hline
\end{tabular}

Fonte: Investigación del autor. 


\section{Cuadro 4 - Normativa sobre la educación ambiental en Colombia}

\begin{tabular}{|l|}
\hline Secretaría Distrital de Educación y Secretaría Distrital de Ambiente (2007).Decreto \\
Distrital 617: Politica Pública Distrital de Educación Ambiental. En: <http://www. \\
secretariadeambiente.gov.co/sda/libreria/pdf/politica_educacion_ambiental.pdf> \\
\hline Ministerio del Medio Ambiente y Ministerio de Educación Nacional (2002). Política \\
Nacional de Educación Ambiental, SINA.En: <http://www.minambiente.gov.co// \\
documentos/1421_Política_Nacional_de_Educaicón_Ambiental.pdf> \\
\hline $\begin{array}{l}\text { Ministerio de Educación Nacional (1994). Decreto 1743, por el cual se instituye el Proyecto de } \\
\text { Educación Ambiental para todos los niveles de educación formal. Diario Oficial, n. 41.476. En: } \\
\text { <http://www.presidencia.gov.co/prensa_new/decretoslinea/1994/agosto/03/dec1743031994.pdf> }\end{array}$ \\
\hline
\end{tabular}

Fonte: Investigación del autor.

\subsection{Selección de artículos para el análisis por contenido}

Finalmente, del total de los artículos que hacen referencia a la relación entre las palabras clave, sólo se seleccionan treinta para ser analizados, en función de su contenido y aporte al objetivo específico de esta investigación en tanto conceptualizan respecto a la pedagogía y la didáctica ambiental; los demás documentos sí poseen las palabras clave, pero su contenido no es significativo para los intereses del estudio. A continuación se detalla sobre los artículos seleccionados.

Cuadro 5 - Análisis bibliométrico de los treinta artículos seleccionados

\begin{tabular}{|l|l|}
\hline Artículos en español & 11 \\
\hline Artículos en inglés & 13 \\
\hline Artículos en portugués & 6 \\
\hline Casos en que los autores son de & $\begin{array}{l}\text { Colombia, 10; Brasil, 7; España, 3; Estados Unidos 3; } \\
\text { Turquía, 3; Taiwán, 2; Nigeria 1; Botsuana, 1; Grecia, 1; } \\
\text { Canadá, 1; Nueva Zelanda, 1; Finlandia, 1; Portugal, 1. }\end{array}$ \\
\hline Artículos por revista & $\begin{array}{l}\text { International Journal of Environmental and Science Education } \\
\text { (Int.), 13; Revista Luna Azul (Col.), 8; Revista Ciência } \\
\text { em Extensão (Bra.), 5; Revista Eureka sobre Enseñanza y } \\
\text { Divulgación de las Ciencias (Esp.), 2; Sociedade \& Natureza } \\
\text { (Bra.), 1; Revista Ibero Americana de Educación (Int.), 1. }\end{array}$ \\
\hline Año de publicación & $\begin{array}{l}\text { 2009, 9; 2007, 6; 2010, 5; 2008, 3; 2011, 3; } \\
\text { 2004, 1;2005, 1; 2006, 1; 2012, 1. }\end{array}$ \\
\hline
\end{tabular}

Fonte: Investigación del autor.

\section{ANÁLISIS DE LA INFORMACIÓN}

\subsection{Identificación de los fundamentos pedagógicos y didácticos en cada artículo}

Posterior al trabajo de búsqueda de fuentes, se construyó un listado de referencias por su título y resumen, que potencialmente aportan a definir las categorías "pedagogía ambiental"y "didáctica ambiental". De esta manera se extrae textualmente de cada uno de los treinta artículos aquello que apunta a lo pedagógico y a lo didáctico. 


\subsection{Análisis de tendencias en cuanto a lo pedagógico ambiental y didáctico ambiental}

El análisis del contenido de los treinta artículos, como se señaló anteriormente, permite hacer agrupaciones, de tal manera que se establecen algunas tendencias en cuanto a la forma de concebir lo que puede enunciarse como pedagogía ambiental y didáctica ambiental. Antes de proceder a señalar los enfoques o tendencias, es importante señalar varios aspectos referentes a lo hallado:

- A pesar de que la búsqueda por la base de datos permite filtrar artículos que contengan las palabras clave y los títulos sugieran que hacen un aporte sobre la temática que ocupa esta investigación, por su contenido muchos de los artículos no aportan para los objetivos buscados; esto justifica la reducción de la lista inicial a los treinta artículos seleccionados. Así mismo, dentro de los últimos artículos seleccionados, no todos hacen referencia a la educación ambiental en la educación superior; siendo más frecuente encontrar artículos con importantes aportes para la construcción de lo pedagógico y lo didáctico ambiental, ubicados en el contexto de la educación superior, y en menor medida, en la educación ciudadana o de comunidades.

- En los artículos revisados, no hay mención explícita de la "pedagogía ambiental", ni de la "didáctica ambiental"; por lo que se hace el análisis del contenido a partir de la conceptualización de las categorías definidas en el primer numeral de la metodología de este artículo.

- En varios casos se encuentra que los artículos proveen elementos conceptuales que se acercan a su visión de lo que puede ser la pedagogía ambiental y/o la didáctica ambiental. Esto quiere decir que algunos artículos aportan a ambas categorías, pero que otros sólo aportan de manera suficiente a una de las dos categorías; por ejemplo, Lizmova (2007) no presenta fundamentos en torno al tema pedagógico, sino que se centra en presentar cómo la cartografía aporta a la comprensión de tópicos en lo ambiental, y así mismo, Rivera (2006) y Bonil et al. (2010) no presentan fundamentos ni experiencias en torno al tema didáctico, puesto que el principal objetivo de sus artículos es fundamentar la educación ambiental desde la complejidad.

\subsubsection{Tendencias respecto a la concepción de la pedagogía ambiental}

\section{a. Tendencia hacia la formación en la conciencia, el cuidado y preservación de lo natural}

Esta tendencia se caracteriza porque hay un fuerte enfoque en el que se conciben y estructuran los procesos educativos en función de lograr conciencia o cambio de actitudes en los sujetos, para que éstos le den mayor valor a lo natural y se comprometan con su cuidado o conservación; muestra de ellos son las propuestas 
dirigidas a comunidades o público en general (Kanevieskir et al., 2009; Reis et al., 2007). Estudios como los de Ospina y Murcia (2007) muestran cómo, en la universidad, hay una tendencia a seguir separando lo "natural" y lo "humano", lo que de alguna manera conlleva a la idea de que el hombre debe "volver a lo natural"; siendo ésta en últimas una concepción "natural o verde" del ambiente.

En la búsqueda de esos cambios de actitudes y valores, adquisición de conocimientos, desarrollo de habilidades y/o toma de conciencia, es notable ver que se proponen que los perfiles de los "sujetos responsables con el ambiente" se forman cuando los procesos educativos se desarrollan en espacios alternativos a la escuela, en los que los sujetos tendrán contacto directo con aquello que deben preservar (Dimopoulos et al., 2009; Jeronen et al., 2009; Ramos, I. et al., 2009), tales como parques, jardines botánicos, reservas naturales, zoológicos.

Con la revisión de los referentes, es posible encontrar posturas sobre la formación en lo ambiental que se enfocan hacia el cuidado y preservación de lo natural desde lo específico de las clases de ciencias naturales, como es el caso de V. Ramos et al. (2009), que abordan el problema desde la alfabetización científica. Así mismo, es posible ver que no todas las propuestas que hacen referencia a el cuidado y preservación se limitan a lo saber, lo reflexivo y la actitud del sujeto, sino que también lo abordan desde la concepción de lo público y desde lo político (Piroli; Ramos, 2010).

Con lo anterior puede inferirse que esta forma de asumir la pedagogía ambiental, significa que:

a) la educación es un proceso de toma de conciencia, cambio de actitudes y valores, adquisición de conocimientos y desarrollo de habilidades, dirigido a la protección y cuidado del ambiente; siendo algo que se consigue notablemente en los mismos contextos naturales;

b) quienes orientan los procesos educativos son quienes facilitan la aproximación del sujeto a lo natural y son quienes disponen recursos y saberes para los aprendizajes;

c) el currículo que orienta la formación del sujeto se fundamenta en experiencias y saberes en torno a lo natural y las implicaciones de las acciones humanas sobre ello.

b. Tendencia hacia la formación en una concepción y acción compleja del ambiente

Esta tendencia se identifica porque existen múltiples propuestas que fundamentan lo que puede entenderse como pedagogía ambiental tendiendo como principales supuestos:

a) el ambiente es complejo, por lo que comprende relaciones entre las esferas social (desde lo humano, ético, político, educativo, cultural etc.) y lo biofísico (especies, atmósfera, litósfera etc. y sus relaciones); 
b) la educación busca formar sujetos en la multidimensionalidad de su ser, que sean capaces de hacer una lectura compleja del ambiente y así mismo de proponer acciones complejas sobre el ambiente, para su transformación;

c) los procesos educativos se estructuran de acuerdo a los contextos ambientales particulares, por lo que son flexibles;

d) se busca hacer integración de saberes y experiencias, de tal manera que se construya esa postura compleja;

e) se busca que los sujetos aporten a la formación ambiental compleja de otros. Así mismo, la revisión y análisis de la literatura permiten identificar dos grandes enfoques dentro de esta tendencia:

- Procesos en la formación en ciencias: partiendo de la necesidad de hacer una lectura del ambiente, se llega a comprender la relevancia de los impactos o consecuencias que tiene el desarrollo o aplicación del conocimiento científico y tecnológico en las esferas sociales y ambientales, por lo que se llega a plantear procesos educativos que se dirijan a la formación de sujetos que hagan una interpretación de las relaciones entre ciencia, tecnología, sociedad y ambiente, busquen el equilibrio de dichas relaciones, cambien sus actitudes, establezcan nuevas perspectivas éticas, busquen la solución de situaciones problema y participen como ciudadanos en los procesos de toma de decisiones o políticas públicas sobre el uso y desarrollo de la CyT (Ikbal; Kaptan, 2008; Steele, 2011; Su, 2011). En general, varios de los referentes en esta postura buscan que el conocimiento científico sea visto como una alternativa para solucionar los problemas ambientales, por lo que buscan dar un contextos social y ambiental específico a las teorías científicas y a los procesos tecnológicos, así como introducir el estudio de las relaciones CTSA a todo el currículo de ciencias (Carvalho-Knighton; Smoak, 2009; Conde; Sánchez, 2010; Parlo; Butler, 2007; Raper; Zander, 2009; $\mathrm{Su}, 2011)$.

- Procesos curriculares generales: estudios respecto a la literatura especializada en los temas educativos ambientales identifican una importante tendencia hacia dar relevancia a la dimensión social de la llamada problemática ambiental, así como a la necesidad de una lectura e intervención desde lo interdisciplinar, el cambio de actitudes y valores, así como la búsqueda de posibles soluciones a las situaciones problema (Ospina; Murcia, 2007).

Las posturas agrupadas en esta tendencia conciben los procesos educativos dinámicos, los cuales se estructuran desde los contextos ambientales específicos y los cuales se transforman en función de los cambios en ese contexto; así mismo buscan la inclusión de todos los actores de la comunidad y no sólo buscan la formación en el saber respecto a los ecosistemas e impactos negativos de las acciones humanas, sino que buscan la formación política, económica, social, intercultural, ética, tecnológica, 
estética, humana, creativa, organizativa, estratégica, interdisciplinar (Doğru, 2008; Sepúlveda, 2009; 2010; Tovar-Gálvez, 2011; Vega et al.,2007; Yue et al.,2010).Todo lo enunciado significa cambios radicales en los currículos y en la administración de las instituciones (Ramírez; Sepúlveda, 2007), puesto que significa reconocer nuevas relaciones entre los componentes de ese complejo ambiente (Rivera, 2006).

Por lo general, los procesos curriculares orientados desde este enfoque buscan estructurar y desarrollar los procesos desde el reconocimiento de las comunidades en sus contextos, a partir de diagnósticos que permiten identificar los aspectos relevantes a estudiar y a transformar en la cultura, en lo personal o en las formas de vivir (Kılınç, 2010; Molano, 2006); se buscan la planeación de experiencias que permitan el reconocimiento de las situaciones problema, la participación y la transformación de esos contextos, siendo ésta otra forma de aproximarse a la realidad para construir modelos para comprenderla e intervenirla (Ajiboye; Ajitoni, 2008; Bonil et al., 2010; Doğru, 2008; Tovar-Gálvez, 2012); también siendo relevante la propuesta de buscar formar a los sujetos para que a su vez aporten a la formación ambiental de otros en sus grupos, familias y comunidades (Freitas; Muno, 2005; Tovar-Gálvez, 2012).

\subsubsection{Tendencias respecto a la concepción de la didáctica ambiental}

\section{a. Tendencia hacia las actividades sin articulación o fundamento}

En esta tendencia hacia asumir los procesos sin fundamentos claros o estructurados sobre enseñanza-aprendizaje-evaluación, se encuentran propuestas en las que los "aprendizajes" o la "enseñanza" de aquello que logra la "formación ambiental" de los sujetos se centran en metodologías constituidas por varias actividades dirigidas a comunidades, tales como uso de videos, periódicos murales, carteleras, títeres, informativos, dramatizaciones y de los llamados "materiales didácticos" que buscan lo lúdico y agradable para las personas, cursos, charlas, talleres, ilustraciones, modelos, imágenes, caminatas, visitas a lugares alternos a la escuela, música etc., como lo muestra un amplio estudio de Ospina y Murcia (2007) y como se evidencia en las propuestas de Reis et al., (2007), V. Ramos et al. (2009), Jeronen et al., (2009), I. Ramos et al. (2009), Kanevieskir et al. (2009) y Piroli y Ramos (2010).

Así esta forma de asumir la didáctica ambiental se caracteriza por: a) centrarse principalmente en la experiencia; b) se busca la motivación y la atención de los sujetos hacia los temas a tratar; c) las actividades son múltiples y no necesariamente están articuladas u organizadas según un fundamento teórico sobre la enseñanza-aprendizaje-evaluación.

\section{b. Tendencia hacia el estudio de casos y resolución de problemas desde el aula}

Esta tendencia se caracteriza porque las propuestas que la alimentan plantean el aprendizaje-enseñanza-evaluación desde la resolución de problemas y el estudio de casos específicos, lo que supone que logra integrar los saberes, actitudes y habilidades de los estudiantes en torno a las situaciones ambientales que se plantean 
como ejes o núcleos en los espacios curriculares (Dimopoulos et al., 2009; Doğru 2008; Ramírez; Sepúlveda, 2007) para lo que se proponen metodologías particulares, en ocasiones dirigidas hacia determinadas temáticas, como el caso de Lizmova (2007) quien argumenta cómo desde el método cartográfico se logra hacer una lectura y comprensión de varias dimensiones del ambiente, u otros casos como la implementación de experimentos y simulaciones sobre situaciones ambientales a través de las Tecnologías Digitales ( $\mathrm{Su}, 2011$ ), la articulación de la paleo-ecología al currículo (Raper; Zander, 2009), el estudio del problema de la calidad del agua y su comprensión-solución a través del laboratorio de análisis de ciencias ambientales y el uso de tecnologías informáticas (Carvalho-Knighton; Smoak, 2009).

Así, esta concepción de la didáctica ambiental centrada en la resolución de problemas, estudio de casos y desarrollo de talleres, se caracteriza por:

a) asumir el aprendizaje en términos de lo conceptual, lo metodológico, las actitudes y lo tecnológico;

b) la enseñanza busca que el sujeto integre las diferentes dimensiones del saber en la resolución del problema o estudio del caso;

c) se da un contexto específico al conocimiento;

d) las metodologías son variadas y por lo general incluye la dimensión experimental en el marco de la solución del problema;

e) esta tendencia es propia de la enseñanza de las ciencias con enfoque ambiental.

\section{c. Tendencia hacia la formación por proyectos}

Esta tendencia se constituye por propuestas didácticas sustentadas principalmente desde elementos constructivistas y de la complejidad, asumiendo que la enseñanza-aprendizaje-evaluación es un proceso que se da de manera contextualizada a través de proyectos que promueven articular las múltiples dimensiones del ser, los diferentes actores y los recursos, con el fin de transformar realidades ambientales; así, lo proyectos se estructuran desde los contextos, son inclusivos, buscan la articulación curricular, busca la formación política y la participación ciudadana, buscan la lectura y acción compleja del ambiente, involucran aspectos conceptuales, procedimentales, valorativos y emotivos de los sujetos, promueven la investigación-acción y buscan la articulación de las instituciones con su entorno (Ajiboye; Ajitoni, 2008; Freitas; Muno 2005; Kilinç, 2010; Molano, 2006; Sepúlveda, 2009; 2010; Steele, 2011; Tovar-Gálvez, 2011; Vega, et al., 2007; Yue et al., 2010).

Algunas propuestas de trabajo por proyectos e ubican en temas específicos de las ciencias y lo ambiental, tales como Kilınç (2010), quien los ubica en la didáctica de las ciencias naturales, Conde y Sánchez (2010), quienes plantean el programa de eco-escuelas en las que los estudiantes realizan proyectos de eco-auditorias, Parlo y Butler (2007), quienes desarrollan un programa de formación ambiental de docentes a través del proyecto "de los ríos a los arrecifes", y como Ikbal y Kaptan 
(2008), quienes incursionan en proyectos a través de los cuales los estudiantes pueden evidenciar cómo las aplicaciones de la ciencia y la tecnología pueden afectar a las personas, la sociedad y el ambiente.

Por lo general, las metodologías para trabajar por proyectos incluyen una contextualización o diagnóstico en lo que se reconoce el sujeto, la comunidad y el territorio, la definición de las situaciones ambientales prioritarias o problema y se diseñan planes de acción-gestión ambiental, la implementación de las estrategias, la evaluación de los resultados, así como programas de educación comunitaria y establecimiento de nuevos procesos (Tovar-Gálvez, 2012; Vega et al., 2007).

En general, esta concepción sobre didáctica ambiental tiene como supuestos:

a) el ambiente es complejo y en esa medida, la acción didáctica es compleja;

b) la enseñanza-aprendizaje-evaluación abarca múltiples dimensiones del saber, del ser y del contexto ambiental;

c) los proyectos son el medio a través del cual se hace la formación del sujeto y a través del cual se logra la transformación de la realidad;

d) los proyectos son procesos dinámicos.

\section{CONCLUSIONES}

El tema del currículo es amplio y complejo; frente a lo cual al menos se requiere una fundamentación pedagógica y didáctica, sin decir que ello sea lo único a tener en cuenta. Ahora bien, para llegar a la fundamentación del currículo para la formación ambiental en la educación superior es necesario construir una postura sobre la pedagogía ambiental y la didáctica ambiental; lo que justifica hacer una primera revisión de la literatura especializada y a través del análisis de los contenidos identificar las tendencias teórico-prácticas que sobre dichos conceptos se han venido configurando.

La construcción del estado del arte evidencia que la literatura aborda el tema de la educación ambiental de manera amplia, pero que no hay elaboraciones precisas sobre los conceptos pedagogía ambiental y la didáctica ambiental; aspecto que obliga a pensar un mecanismo para desentrañar las concepciones sobre pedagogía ambiental y la didáctica ambiental que están en el trasfondo de las reflexiones y experiencias revisadas. Así, la conceptualización de lo pedagógico y lo didáctico, de manera general, permite identificar unos ejes, cuestionamiento o aspectos que les definen, y de allí surgen los criterios de análisis para abordar los referentes identificados.

Con la agrupación de los resultados, para identificar tendencias sobre pedagogía ambiental y la didáctica ambiental de manera inductiva, se halla que existen dos grandes sustentos teóricos: a) uno que se cimenta en la posesión de saberes especializados y en el activismo, para el cual la formación de los sujetos debe ser orientada hacia la conciencia, el cuidado y la preservación, lo que se construye a través del estudio de las disciplinas y en la variedad de experiencias; b) y otro que se 
instituye en una visión y acción compleja sobre el ambiente, para el cual la formación de los sujetos es una construcción desde los contextos, lo que se logra a través de procesos en los que se estudia e intervienen los contextos desde diversas alternativas.

Finalmente, el presente estudio aporta al campo de investigación en tanto muestra un panorama en cuanto a las formas de entender la pedagogía ambiental y la didáctica ambiental; conduciendo así a la posibilidad de asumir otra tendencia o a quizás a proponer alternativas ante las posibles limitaciones de lo identificado. Sin embargo, el camino de la construcción de un currículo para la formación ambiental para la educación ambiental es un campo que sigue abierto, amplio y complejo, pues quedan muchas preguntas como: ‘además de lo pedagógico y lo didáctico, qué otras dimensiones fundamentan un currículo para la formación ambiental?, ¿qué concepción de universidad, de formación profesional y de docencia universitaria significan unas u otras concepciones sobre pedagogía ambiental y sobre didáctica ambiental?, ¿qué trasfondos filosóficos y epistemológicos están de trasfondo de una u otra versión sobre la pedagogía ambiental y sobre la didáctica ambiental?, entre muchas otras.

\section{REFERENCIAS}

Adúriz-Bravo, Agustín; IzQuierdo, Mercè. Acerca de la didáctica de las ciencias como disciplina autónoma. Revista Electrónica de Enseñanza de las Ciencias, Vigo: Universidad de Vigo; Educación Editora, v. 1, n. 3, p. 130-140, 2002. Disponible en: <http://www. saum.uvigo.es/reec/volumenes/volumen1/Numero3/Art1.pdf>. Acceso en: 20 ago. 2011.

Aisenberg, Beatriz. Didáctica de las ciencias sociales: ¿desde qué teorías estudiamos la enseñanza? Revista de Teoría y Didáctica de las Ciencias Sociales, Mérida: Universidad de los Andes, v. 3, n. 3, p. 1-28, ene.-dic. 1998. Disponible en: <http://www.saber.ula.ve/ bitstream/123456789/23916/1/bol3_beatriz_aisenberg.pdf>. Acceso en: 20 ago. 2011. Ajiвoye, Josiah; Ajitoni, S. Effects of Full and Quasi - Participatory Learning Strategies on Nigerian Senior Secondary Students' Environmental Knowledge: Implications for Classroom Practice. International Journal of Environmental E Science Education, Bolu: IJESE, v. 3, n. 2, p. 58-66, abr. 2008. Disponible en: <http://www.ijese. com/v3n2_Ajiboye.pdf>. Acceso en: 20 ago. 2011.

Bonil, Joseph; Junyent, María; Pujol, Rosa. Educación para la sostenibilidad desde la perspectiva de la complejidad. Revista Eureka sobre Enseñanza y Divulgación de la Ciencia, Cádiz: Universidad de Cádiz y Asociación de Profesores de Ciencias, v. 7, n. Extraordinario, p. 198-215, feb. 2010. Disponible en: <http://reuredc.uca.es/index.php/ tavira/article/viewFile/40/39>. Acceso en: 20 ago. 2011.

Cachapuz, Antonio; Praia, Joao; Gil-Pérez, Daniel; Carrascosa, Jaime; Martínez, Isabel. A emergência da didáctica das ciências como campo específico de conhecimento. Revista Portuguesa de Educação, Braga: Universidade do Minho; CEEP, v. 14, n. 1, p. 155-195, 2001. Disponible en: <http://redalyc.uaemex.mx/pdf/374/37414108.pdf>. Acceso en: 20 ago. 2011. 
Carvalho-Knighton, Kathleen; Smoak, Joseph. Integrating basic analytical methods and computer-interface technology into an environmental science water quality lab improves student attitude. International Journal of Environmental E Science Education, Bolu: IJese, v. 4, n. 4, p. 419-428, oct. 2009. Disponible en: <http://www.ijese.com/ IJESE_v4n4_Carvalho-Knighton.pdf>. Acceso en: 20 ago. 2011.

Conde, María; SÁNchez, Samuel. The school curriculum and environmental education: A school environmental audit experience. International Journal of Environmental E Science Education, Bolu: IJESE, v. 5, n. 4, p. 477-494, oct. 2010. Disponible en: <http:// www.ijese.com/IJESE_v5n4_Conde_and_Sanchez.pdf>. Acceso en: 20 ago. 2011.

Díaz, Joaquín. Didáctica de las lenguas extranjeras: los enfoques comunicativos. Didáctica (Lengua y Literatura), Madrid: Universidad Complutense de Madrid; Servicio de Publicaciones UCM, v. 8, p. 87-103, 1996. Disponible en: <http://revistas.ucm.es/ index.php/DIDA/article/view/DIDA9696110087A>. Acceso en: 20 ago. 2011.

DíAz, Víctor. Teoría emergente en la construcción del saber pedagógico. Revista Iberoamericana de Educación, Madrid: OEI, v. 37, n. 3, p. 1-19, dic. 2005. Disponible en: <http://www.rieoei.org/deloslectores/1122Diaz.pdf〉. Acceso en: ago. 2011.

Dimopoulos, Dimitrios; Paras kevopoulos, Stefanos; Pantis, John. Planning educational activities and teaching strategies on constructing a conservation educational module. International Journal of Environmental E' Science Education, Bolu: IJese, v. 4, n. 4, p. 351-264, oct. 2009. Disponible en: <http://www.ijese.com/IJESE_v4n4_Dimopoulos. pdf>. Acceso en: 20 ago. 2011.

DoĞRu, Mustafa. The application of problem solving method on science teacher trainees on the solution of the environmental problems. International Journal of Environmental E Science Education, Bolu: IJEse, v. 3, n. 1, p. 9-18, ene. 2008. Disponible en: <http:// www.ijese.com/V3_N1_Dogru.pdf >. Acceso en: 20 ago. 2011.

Ferrández, Antonio. Las teorías del currículum. In: Medina, Antonio; Sevillano, Luisa (Coords.). El currículum: fundamentos, diseño, desarrollo y evaluación. Madrid: UNED, 1991.

Fernández, Gabriela. Pedagogía, psicología y didáctica de la matemática. Docencia de Matemáticas en la Economía y la Empresa, Sevilla: ASEPUMA, 2000. Disponible en: $<$ http://150.214.55.100/asepuma/sevilla2000/m3-01.pdf>. Acceso en: 20 ago. 2011.

Freitas, Valeria; Muno, Marlene. A pedagogia de projetos na práxis da educação ambiental: uma experiência na Escola Agrotécnica Federal de Uberlândia, MG, 2003-2004. Sociedade e Natureza, Uberlândia: Universidade Federal de Uberlândia, v. 17, n. 32, p. 133-145, jun. 2005. Disponible en: <http://www.seer.ufu.br/index.php/ sociedadenatureza/article/viewFile/9171/5640 > . Accesso em: 20 ago. 2011.

Gallego-Badillo, Rómulo. Un concepto epistemológico de modelo para la didáctica de las ciencias experimentales. Revista Electrónica de Enseñanza de las Ciencias, Vigo: Universidad de Vigo; Educación Editora, v. 3, n. 3, p. 301-319, 2004. Disponible en: $<$ http://www.saum.uvigo.es/reec/volumenes/volumen3/Numero3/ART4_VOL3_ N3.pdf>. Acceso en: 20 ago. 2011. 
Godino, Juan. Perspectiva de la didáctica de las matemáticas como disciplina tecnocientifica. Granada: Universidad de Granada, 2010. Disponible en: <http://www.ugr.es/ jgodino/ fundamentos_teoricos/perspectiva_ddm.pdf $>$. Acceso en: 20 ago. 2011.

. Indicadores de la idoneidad didáctica de procesos de enseñanza y aprendizaje de las matemáticas. Conferencia Interamericana de Educación Matemática, 13., 2011. Recife. Anais... : Comité Interamericano de Educación Matemática, 2011. Disponible en: <http://www.ugr.es/ jggodino/eos/jdgodino_indicadores_idoneidad. pdf>. Acceso en: 20 ago. 2011.

Grupo Conciencia. Hacia un curriculo para la formación ambiental en la educación superior colombiana. [Documento de Trabajo]. Bogotá: Universidad Antonio Nariño, 2011.

Guerra, Jorge. Proceso de diseño curricular. Medellín: Fundación Universitaria Luis Amigó, 2008. Disponible en: <http://www.postgrado.unesr.edu.ve/acontece/es/ todosnumeros/num13/02_06/procesodeldisenocurricular.pdf $>$. Acceso en: 20 ago. 2011. Iквal, Mehmet; Kaptan, Fitnat. STS from a historical perspective and its reflection on the curricula in Turkey. International Journal of Environmental \& Science Education, Bolu: IJEse, v. 3, n. 1, p. 3-8, ene. 2008. Disponible en: <http://www.ijese.com/V3_N1_ Yetisir_Kaptan.pdf>.Accesso en: 20 ago. 2011.

Jeronen, Eila; Jeronen, Juha; Raustia, Hanna. Environmental education in Finland: a case study of environmental education in nature schools. International Journal of Environmental E Science Education, Bolu: IJESE, v. 4, n. 1, p. 1-23, ene. 2009. Disponible en: <http://www.ijese.com/IJESE_v4n1_Jeronen\%20et\%20al..pdf>. Acceso en: 20 ago. 2011.

Kanevieskir, Thiciany; Garcia, Yara; Santos, Eder dos; Carvalho, María; Leal, Antonio; Guimarães, Eliana. Valorizando a água e o lixo na educação ambiental. Reviista Ciência em Extensão, São Paulo: Pró-Reitoria de Extensão Universitária-UNESP, v. 5, n. 1, p. 74-87, 2009. Disponible en: <http://ojs.unesp.br/index.php/revista_proex/article/ view/313>. Acceso en: 20 ago. 2011.

KILInç, Ahmet. Can project-based learning close the gap? Turkish student teachers and proenvironmental behaviours. International Journal of Environmental Es Science Education, Bolu: IJese, v. 5, n. 4, p. 495-510, oct. 2010. Disponible en: http://www.ijese. com/IJESE_v5n4_Kilinc.pdf. Acceso en: ago. 2011.

Lizmova, Nina. Análisis de mapas como un método de investigación de fenómenos naturales y socioeconómicos. Luna Azul, Manizales: Universidad de Caldas, v. 24, p. 74-80, ene.-jun. 2007. Disponible en: <http://lunazul.ucaldas.edu.co/downloads/ Revista24_10.pdf>. Acceso en: 20 ago. 2011.

Molano, Carlos. Desarrollo de la conciencia ambiental por medio de la lúdica. Una propuesta pedagógica desde la educación ambiental para el desarrollo rural. Luna Azul, Manizales:Universidad de Caldas,v.18,p.1-19, ene.-jun.2006.Disponible en: <http://lunazul. ucaldas.edu.co/index.php?option $=$ com_content\&task=view\&id $=65 \& I$ temid $=65>$. Acceso en: ago. 2011. 
Moreno, María. De la matemática formal a la matemática escolar. $P N A$, Granada: Universidad de Granada, v. 1, n. 3, p. 99-111, 2007. Disponible en: <http://www.pna. es/Numeros2/pdf/Moreno2007De.pdf>. Acceso en: 20 ago. 2011.

Núñez, María; Fernández, Eduardo; Romero, Antonio. Research in language didactics: a model for the evaluation of oral discursive competence in secondary education. Porta Linguarum, Granada: Universidad de Granada, v. 9, p. 111-126, ene. 2008. Disponible en: <http://www.ugr.es/ portalin/articulos/PL_numero9/8\%20 Pilar\%20Nunez.pdf>. Acceso en: ago. 2011.

Ospina, Luz; Murcia, Napoleón. Imaginarios ambientales que irradian la universidad de Caldas.Luna Azul,Manizales:Universidad de Caldas,v.25,p.39-60,jul.-dic.2007.Disponible en: <http://lunazul.ucaldas.edu.co/index.php?option=content\&task=view\&id=352 >. Acceso en: 20 ago. 2011.

Pacheco, José Augusto. Teoria curricular crítica: os dilemas (e contradições) dos educadores críticos. Revista Portuguesa de Educação, Braga: Instituto de Educação da Universidade do Minho, v. 14, n. 001, p. 49-71, 2001. Disponible en: <http://redalyc. uaemex.mx/src/inicio/ArtPdfRed.jsp?iCve=37414104>. Acceso en: 20 ago. 2011.

PAGÉs, Joan. La didáctica de las ciencias sociales, el currículum y la formación del profesorado. Signos teoría y práctica de la educación, Gijón: Centro del Profesorado de Gijón, v. 5, n. 13, p. 38-51, oct.-dic. 1994. Disponible en: <http://www.quadernsdigitals. net/datos_web/hemeroteca/r_3/nr_39/a_617/617.html>. Acceso en: 20 ago. 2011.

PARLo, Amy; Butler, Malcolm. Impediments to Environmental Education Instruction in the Classroom: A Post-Workshop Inquiry. International Journal of Environmental E Science Education, Bolu: IJEsE, v. 2, n. 1, p. 32-37, ene. 2007. Disponible en: <http:// www.ijese.com/IJESE_V3_N1_Parlo\&Butler.pdf>. Acceso en: 20 ago. 2011.

Piroli, Edson; Ramos, Vanessa. Educação ambiental aplicada como instrumento de integração universidade-sociedade: experiências em ROSANA-SP. Revista Ciência em Extensão, São Paulo: Pró-Reitoria de Extensão Universitária-UNESP, v. 6, n. 1, p. 138-151, 2010. Disponible en: <http://ojs.unesp.br/index.php/revista_proex/article/ view/340 >. Acceso en: 20 ago. 2011.

PRATS, Joaquim. Disciplinas e interdisciplinariedad: el espacio relacional y polivalente de los contenidos de la didáctica de las ciencias sociales. Iber: Didáctica de las Ciencias Sociales, Geografía e Historia, Barcelona: IRIF-SL, v. 24, p. 7-18, 2000.

. Líneas de investigación en didáctica de las ciencias sociales. História \& Ensino Revista do Laboratório de Ensino de Historiaria/UEL, Barcelona: Universitat de Barcelona, v. 9, p. 1-25, 2003. Disponible en: <http://www.histodidactica.es/CCSS/Lineas-Prats. pdf $>$. Acceso en: 20 ago. 2011.

Ramos, Igor; Carvalho, Edmir; Silva, Renato da. Abordagem ecológica em educação ambiental: elaboração de um guia ilustrado de peixes de água doce. Revista Ciência em Extensão, São Paulo: Pró-Reitoria de Extensão Universitária-UNESP, v. 5, n. 1, p. 74-87, 2009. Disponible en: <http://ojs.unesp.br/index.php/revista_proex/article/view/33/69>. Acceso en: 20 ago. 2011. 
Ramírez, Ginés; Sepúlveda, Luz. Sistematización de la dimensión ambiental en la Universidad de Caldas como un medio para generar un sistema de gestión ambiental de la institución. Luna Azul, Manizales: Universidad de Caldas, v. 25, p. 22-38, jul./ dic. 2007. Disponible en: <http://lunazul.ucaldas.edu.co/downloads/Revista25_3.pdf.> Acceso en: 20 ago. 2011.

Ramos, Vanessa; Correa, Marco; Ragogna, Mariana; Andricioli, Natalia; Righes, Rodrigo. O zoológico como espaço integrador de posturas em educação ambiental. Revista Ciência em Extensão, São Paulo: Pró-Reitoria de Extensão Universitária-UNESP, v. 5, n. 1, p. 119-125, 2009. Disponible en: <http://ojs.unesp.br/index.php/revista_proex/ article/view/66/70>. Acceso en: 20 ago. 2011.

Raper, Diana; Zander, Holli. Paleoecology: An Untapped Resource for Teaching Environmental Change. International Journal of Environmental \& Science Education, Bolu: IJesE, v. 4, n. 4, p. 441-447, oct. 2009. Disponible en: <http://www.ijese.com/ IJESE_v4n4_Raper.pdf >. Acceso en: 20 ago. 2011.

Reis, Débora dos; Leal, Ântônio; Guimarães, Eliana; Balbin, Michele; Pereira, Katia; Pereira, Eder; Matos, Rubens. Educação ambiental na universidade: um diálogo com a comunidade. Revista Ciência em Extensão, São Paulo: Pró-Reitoria de Extensão Universitária-UNESP,v. 3, p. 32, 2007. Disponible en: <http://ojs.unesp.br/index.php/ revista_proex/article/view/225/136>. Acceso en: 20 ago. 2011.

Rivera, Jorge. El papel de la geografía en el estudio de la relación sociedad-naturaleza. Luna Azul, Manizales: Universidad de Caldas, v. 23, p. 23-27, jul.-dic. 2006. Disponible en: <http://lunazul.ucaldas.edu.co/downloads/dee4a6f9Revista23_5.pdf >. Acceso en: 20 ago. 2011.

Román, Martiniano; Díez, Eloisa. Aprendizaje y currículum: diseños curriculares aplicados. Buenos Aires: Novedades Educativas, 2003.

Sepúlveda, Luz. Una evaluación de los procesos educativo-ambientales de Manizales. Luna Azul, Manizales, Universidad de Caldas, v. 28, p. 46-56, ene.-jun. 2009. Disponible en: <http://200.21.104.25/lunazul/downloads/Lunazul28_6.pdf>. Acceso en: 20 ago. 2011.

Sepúlveda, Luz. Praeizar el proyecto educativo institucional: una alternativa para incluir la dimensión ambiental en la educación básica y media. Luna Azul, Manizales: Universidad de Caldas, v. 30, n., 142-163, ene.-jun. 2010. Disponible en: $<$ http://200.21.104.25/lunazul/downloads/Lunazul30_08.pdf>. Acceso en: 20 ago. 2011.

Steele, Astrid. Beyond contradiction: Exploring the work of secondary science teachers as they embed environmental education in curricula. International Journal of Environmental E Science Education, Bolu, IJEse, v. 6, n. 1, p. 1-22, ene. 2011. Disponible en: <http://www.ijese.com/IJESE_v6n1_Steele.pdf〉. Acceso en: 20 ago. 2011.

Su, King-Dow. An intensive ICT-integrated environmental learning strategy for enhancing student performance. International Journal of Environmental \& Science 
Education, Bolu: IJEse, v. 6, n. 1, p. 39-58, ene. 2011. Disponible en: <http://www.ijese. com/IJESE_v6n1_Su.pdf>. Acceso en: 20 ago. 2011.

Tovar-Gálvez, Julio César. Propuesta de modelo de evaluación multidimensional de los aprendizajes en ciencias naturales y su relación con la estructura de la didáctica de las ciencias. Revista Eureka Sobre Enseñanza y Divulgación de las Ciencias, Cádiz: Universidad de Cádiz; Asociación de Profesores de Ciencias, v. 5, n. 3, p. 259273, sept. 2008. Disponible en: <http://redalyc.uaemex.mx/src/inicio/ArtPdfRed. jsp?iCve=92050302 $>$. Acceso en: 20 ago. 2011.

. Propuesta para el aprendizaje del concepto reacción química y generación de actitudes hacia la ciencia, a través del estudio de los problemas de la organización espacial del laboratorio y el manejo de residuos químicos. Revista Electrónica de Enseñanza de las Ciencias, Vigo: Universidad de Vigo; Educación Editora, v. 8, n. 2, p. 490-504, 2009. Disponible en: <http://www.saum.uvigo.es/reec/volumenes/volumen8/ ART6_Vol8_N2.pdf>.Acceso en: 20 ago. 2011.

. La reflexión y la auto-evaluación en la transformación de los procesos de educación ambiental: estudio de un caso en el Jardín Botánico de Bogotá. Luna Azul, Manizales: Universidad de Caldas, v. 32, p. 32-44, ene.-jun. 2011. Disponible en: $<$ http://200.21.104.25/lunazul/downloads/Lunazul32_02.pdf>. Acceso en: 20 ago. 2011.

Hacia una educación ambiental ciudadana contextualizada: consideraciones teóricas y metodológicas desde el trabajo por proyectos. Revista Iberoamericana de Educación, Madrid: OEI, v. 58, n. 2, p. 1-11, mar. 2012. Disponible en: <http://www. rieoei.org/expe/4322Tovar.pdf>. Acceso en: 5 jun. 2012.

; GARCíA, Germán. Investigación en la práctica docente universitaria: obstáculos epistemológicos y alternativas desde la didáctica general constructivista. Educação e Pesquisa, São Paulo: Faculdade de Educação da Universidade de São Paulo, v. 38, n. 4, p. 881-895, oct.-dic. 2012. Disponible en: <http://www.scielo.br/pdf/ep/v38n4/07. pdf $>$. Acceso en: 10 dic. 2012.

Trujillo, Fernando. En torno a la interculturalidad: reflexiones sobre cultura y comunicación para la didáctica de la lengua. Porta Linguarum, Granada: Universidad de Granada, v. 4, p. 23-39, jun. 2005. Disponible en: <http://www.ugr.es/ portalin/ articulos/PL_numero4/trujillo.pdf >. Acceso en: 20 ago. 2011.

Vega, Pedro; Freitas, Mario; Álvarez, Pablo; Fleuri, Reinaldo. Marco teórico y metodológico de educación ambiental e intercultural para un desarrollo sostenible. Revista Eureka sobre Enseñanza y Divulgación de la Ciencia, Cádiz: Universidad de Cádiz; Asociación de Profesores de Ciencias, v. 4, n. 3, p. 539-554, 2007. Disponible en: $<\mathrm{http} / /$ venus.uca.es/eureka/revista/Volumen4/Numero_4_3/VegaMarcote_et_al_2007. pdf>. Acceso en: 20 ago. 2011.

Velázquez, Isabel. Una aproximación al mapa disciplinar de la pedagogía. Revista Iberoamericana de Educación, Madrid: OEI, v. 35, n. 2, p.1-16, feb. 2005. Disponible en: $<$ http://www.rieoei.org/deloslectores/811Velazquez.PDF>. Acceso en: 20 ago. 2011. 
Vez, José. La DLE: de hoy para mañana. Porta Linguarum, Granada: Universidad de Granada, v. 1,p. 5-30, ene. 2004.Disponible en: <http://www.ugr.es/ portalin/articulos/ PL_numero1/vez.pdf >. Acceso en: 20 ago. 2011.

Yue, Mei-Chun; Crowie, Bronwen; Barker, Miles; Jones, Alister. What influences the emergence of a new subject in schools? The case of environmental education. International Journal of Environmental \& Science Education, Bolu: IJEse, v. 5, n. 3, p. 265-285, jul.2010. Disponible en: <http://www.ijese.com/IJESE_v5n3_Yueh-et-al. pdf>. Acceso en: 20 ago. 2011.

ZACCAGNINI, Mario. Impacto de los paradigmas pedagógicos históricos en las prácticas educativas contemporáneas. Revista Iberoamericana de Educación, Madrid: OEI, p. 1-30, mayo 2003. Disponible en: <http://www.rieoei.org/deloslectores/443Zaccagnini.pdf〉. Acceso en: 20 ago. 2011.

Zambrano, Armando. Conocimiento, saber y pensamiento: una aproximación a la didáctica de las matemáticas. Educere, Mérida: Universidad de los Andes, v. 8, n. 26, p. 407-413, jul.-sept. 2004. Disponible en: <http://www.saber.ula.ve/bitstream/123456789/19898/2/ articulo15.pdf>. Acceso en: 20 ago. 2011.

ZANón, Javier. Psicolingüística y didáctica de las lenguas: una aproximación histórica y conceptual. MarcoELE, Revista de Didáctica, Barcelona: Instituto Cervantes, v. 5, p. 1-30, 2007. Disponible en: <http://marcoele.com/descargas/5/zanon-psicolinguistica. pdf $>$. Acceso en: 20 ago. 2011.

\section{SOBRE EL AUTOR}

Julio César Tovar-Gálvez es magíster en docencia de la química por la Universidad Pedagógica Nacional (Colombia). Docente-investigador de la Universidad Antonio Nariño (Colombia).

E-mail: joule_tg@yahoo.com

Recebido em dezembro de 2012 Aprovado em fevereiro de 2013 


\section{JULIO CÉSAR TOVAR-GÁLVEZ}

\section{Pedagogía ambiental y didáctica ambiental como fundamentos del currículo para la formación ambiental}

Se trata el problema de la fundamentación del currículo para la formación ambiental en la educación superior, frente a lo cual es necesario construir los conceptos de pedagogía ambiental y de didáctica ambiental. El marco teórico permite definir las categorías "lo pedagógico" y "lo didáctico", a partir de lo cual se analiza la literatura y con ello definir pedagogía ambiental y didáctica ambiental. El estado del arte permite establecer que existen dos sustentos teóricos: a) uno que se cimenta en la posesión de saberes especializados y en el activismo, para el cual la formación de los sujetos debe ser orientada hacia la conciencia, el cuidado y la preservación, lo que se construye a través del estudio de las disciplinas y desde la experiencia; b) y otro que se instituye en una visión y acción compleja sobre el ambiente, para el cual la formación de los sujetos es una construcción desde los contextos, lo que se logra a través de procesos en los que se estudia e intervienen los contextos.

Palabras clave: educación ambiental; pedagogía; didáctica; currículo; educación superior.

\section{Environmental pedagogy and environmental didactic as the environmental education curriculum foundations}

The problem of the foundation of the curriculum for environmental education in bigher education is studied. In this order is necessary to build the concepts of environmental pedagogy and environmental didactic. The theoretical framework is used to define the categories "pedagogy" and "didactic", from which we analyze the literature and thus define environmental pedagogy and environmental didactic. The state of the art allows identify two theoretical underpinnings: a) one that is founded on the possession of specialized knowledge and activism, for which the formation of the subject should be oriented towards awareness, care and preservation, what is built through the study of the disciplines and from experience; $b$ ) and another that establishing a vision and complex action on the environment, for which the formation of the subject is a construction from the contexts, which is accomplished through the processes being studied and are involved contexts.

Keywords: environmental education; pedagogy; didactics; curriculum; higher education.

\section{Pedagogia ambiental e da didática ambiental como fundações do currículo para a educação ambiental}

Trata-se da questão da fundação do currículo para a educação ambiental no ensino superior, perante a qual é necessário construir os conceitos de pedagogia ambiental e de ensino 
ambiental. O referencial teórico é utilizado para definir as categorias de "pedagogia" e "ensinar", e com base nele analisa-se a literatura e, assim, define-se a pedagogia ambiental e da didática ambiental. O estado da arte permite identificar duas bases teóricas: a) uma que se baseia na posse de conhecimento especializado e no ativismo, para o qual a formação do sujeito deve ser orientada para o cuidado, conscientização e preservação, o que é construído pelo estudo das disciplinas e da experiência; b) e outra que estabelece uma visão e uma ação complexa sobre o meio ambiente, para o qual a formação do sujeito é uma construção dos contextos, que é obtida por meio dos processos em estudo e estão envolvidos contextos.

Palavras-chave: educação ambiental; pedagogia; didática; currículo; ensino superior. 